\section{Risk of Sequelae of COVID-19 in Children Cared for by Primary Care Pediatricians}

148 Italian children ( $n=148)$ suspected of and evaluated for COVID-19 infection during the first phase of the pandemic were followed-up for 6 months.During the follow-up period, no difference in the prevalence of new-onset respiratory, dermatological or neurological symptoms, nor in psychological distress,were observed in children who were positive and negative for SARS-CoV-2.

Keywords:Outcome, Psychological distress, Respiratory problems, SARS-CoV-2.

Published online: Nov 29, 2021; PII: S097475591600376

Several studies have described the characteristics of pediatric coronavirus disease 19 (COVID-19) cases [1-3], but few were based in the primary care setting. An observational study was therefore performed with a group of Italian primary care pediatricians with the aim to describe the characteristics of children visited for a suspected severe acute respiratory syndrome 2 (SARS-CoV-2) infection and to monitor their health status in the 6 months after the first visit.

Information concerning children with suspected SARS-CoV-2 infection during the first phase of the pandemic (February, 2020June, 2020) was collected by a group of 35 family pediatricians in the Lombardy Region, Italy, who voluntarily participated in the study. All pediatricians had a long-standing experience in out-ofhospital pediatric practice. Five of them were actively involved in designing the study, and in planning the questionnaire for data collection.

All Information related to demographics, symptoms, presence of any comorbidity, and diagnostic test results (molecular and/or serological test) was collected by using a pre-designed questionnaire. COVID-19 suspected cases were defined as children with flu-like symptoms and children with family members who had a suspected or confirmed COVID-19 infection. Molecular tests (real-time polymerase chain reaction on nasopharyngeal swab) were prescribed by the local health authorities, while serological tests (IgG anti-SARS-CoV-2 detection) were performed based on the parents' decision. Details concerning wheezing episodes, new onset of dermatological (e.g., rash, dermatitis, urticaria) or neurological (e.g., headache, seizures, neuropathies) symptoms, and symptoms potentially associated with psychological distress (e.g., sleep disorders, anxiety, irritability) in the 6-month period after the first consultation were collected by the family pediatricians to monitor the sequelae of the infection. Since the study was mainly descriptive, no a priori sample size calculation was made.

The study was approved by the Fondazione IRCCS Istituto
Neurologico Carlo Besta's Ethics Committee. The characteristics of positive versus negative children were compared using the chisquare test.

Of a total of 349 children were suspected of SARS-CoV-2 infection; 148 (42\%) underwent a diagnostic test and among these $41(28 \%)$ tested positive. Symptom prevalence was slightly higher in SARS-CoV-2 positive children compared to negatives ( $71 \%$ vs $55 \%$ ), with sore throat occurring less commonly in positive children $(18 \%$ vs $51 \%$; $P=0.002)$.

Among those tested positive, three cases had pre-existing comorbidities (asthma, Duchenne muscular dystrophy, and Henoch-Schonlein purpura) as against nine negative cases. Four positive children were hospitalized: two infants (one of whom was an asymptomatic neonate delivered by a positive mother), a 6year-old child, and a 12 year old adolescent. None had pre-existing comorbidities. The length of hospital stay ranged between 5 days to 1 month and, with the exception of the neonate, the hospitalizations were associated with COVID-19 pneumonia. The 12-year-old adolescent developed myocarditis and was admitted to the pediatric intensive care unit for cardiogenic and septic shock.

During the follow-up period of 6 months 107 of the 148 children $(72 \%)$ had contacted or visited the pediatrician for a health issue (range: $1-8$ visits), 38 (26\%) had no health problems, and 3 ( $2 \%$ ) were lost to follow-up. A total of 48 children had come into contact with a positive case during the follow-up. Thirty two of these underwent a molecular test, and only one tested positive (with no previous history of COVID-19).

The prevalence of respiratory, dermatological, and neurological symptoms were similar in positive and negative children during the follow-up period (Table I). In all, 24 children (17\%) had psychological distress, which was not present before the epidemic; 16 developed sleep disorders, 14 anxiety and/or irritability, and 6 both.

To the best of our knowledge, this is the first study describing a series of pediatric COVID-19 cases cared for by primary care pediatricians. Despite the small sample size, some findings are worthy of consideration. During the first pandemic phase, less than half of potentially infected children underwent diagnostic tests, with a prevalence of 1 out of 3 suspected cases. As reported in other studies, fever and cough were the most common symptoms, while in our study a greater proportion of children with malaise were observed [5]. Except sore throat, the most common symptoms were similar in positive and negative children, as observed by Garazzino, et al. [3] in children attending a pediatric hospital for COVID-19.

During the first pandemic phase schools were closed and contagion occurred in the household setting. It is therefore not surprising that the number of potentially affected family members was associated with an increased likelihood of infection incidence. The number of hospitalized children was low; though, the prevalence of moderate-severe cases in children was not negligible. 
Table I Characteristics at Follow-up in Children with Suspected COVID-19 $(N=148)$

\begin{tabular}{|c|c|c|c|}
\hline & $\begin{array}{l}\text { Positive } \\
(N=41)\end{array}$ & $\begin{array}{l}\text { Negative } \\
(N=107)\end{array}$ & $\begin{array}{r}\text { Overall } \\
(N=148)\end{array}$ \\
\hline \multicolumn{4}{|l|}{ Diagnostic test ${ }^{a}$} \\
\hline Molecular & $15(15)$ & $86(85)$ & 101 \\
\hline Serological & $28(38)$ & $45(62)$ & 73 \\
\hline Male gender & $19(26)$ & $55(74)$ & 74 \\
\hline \multicolumn{4}{|l|}{ Age (y) } \\
\hline$<1$ & $3(38)$ & $5(62)$ & 8 \\
\hline $1-5$ & $15(29)$ & $37(71)$ & 52 \\
\hline $6-11$ & $14(22)$ & $49(78)$ & 63 \\
\hline $12-16$ & $9(36)$ & $16(64)$ & 25 \\
\hline Median (IQR) & $7(4-11.5)$ & $6(3-10)$ & $6.5(3.5-10.5)$ \\
\hline \multicolumn{4}{|l|}{ 6-month follow-up } \\
\hline Visits & $1.9(1.7)$ & $2.0(1.3)$ & $2.0(1.4)$ \\
\hline \multicolumn{4}{|l|}{ New-onset symptoms } \\
\hline Wheezing & 0 & $6(6)$ & $6(4)$ \\
\hline Dermatological $^{b}$ & $1(3)$ & $6(6)$ & $7(5)$ \\
\hline Neurological $^{c}$ & $2(5)$ & $5(6)$ & $7(6)$ \\
\hline Psychological distress & $6(15)$ & $18(17)$ & $24(17)$ \\
\hline COVID-19 positive & $0 / 8(20)$ & $1 / 24(23)$ & $32(22)$ \\
\hline
\end{tabular}

Data is reported as no(\%). 2 children were positive to both molecular and serological tests; ${ }^{2} 6$ children underwent both diagnostic tests; ${ }^{b}$ Psoriasis flare $(n=1)$, dermatitis $(n=3)$, rash $(n=2)$, plantar wart $(n=1)$; 'Symptoms due to headache $(n=4)$, Duchenne muscular dystrophy $(n=1)$, Seizure $(n=1)$, not specified $(n=1)$.

No differences between positive and negative children were observed during the follow-up. The sample size may be too small to detect statistically significant differences, but the fact that the proportion in the two groups was very close suggests that a greater likelihood of sequelae in positive cases is unlikely. A few case series have reported and have suggested the occurrence of 'long covid' in the pediatric population $[6,7]$. A comparison with children who tested negative to COVID-19 may be needed, since it is likely that some symptoms may be strongly associated with the pandemic situation and with quarantine, and not only with COVID-19 infection [8]. Given the observational characteristic of the study, no additional diagnostic tests were done and therefore, we were not able to evaluate whether there were any cardiac or central nervous system sequelae in children with no clinical symptoms.

In our sample, psychological distress occurred in nearly 1 in 6 children, consistent with other reports [8-10] with no differences in incidence between positive and negative cases, suggesting that COVID-19 per se is not a risk factor. Despite the limitations of this study, it showed that children with COVID-19 do not seem to be at a greater risk of sequelae than children without.

Acknowledgements: The authors would like to thankthe family paediatricians who participated to the study. Basco Working Group: Patrizia Bardelli, Ketty Bastoni, Sonia Bettini, Angela Biolchini, Jelena Bojanin, Cristina Candiani, Barbara Carli, Maria Angela Cazzuffi, Mariapia De Berti, Chiara Di Francesco, Laura Di Francesco, Lucia Di Maio, Maria Elisabetta Di Pietro, Paola Fornaro,
Paola Maria Gallia, Giuliano Gambarini, Lia Gnemmi, Enrica Heritier, Barbara Loda, Laura Loguercio, Monica Mantegazza, Laura Mauri, Annalisa Monolo, Mario Narducci, Giovannina Pastorelli, Antonella Piazza, Elena Puricelli, Maria Letizia Rabbone, Annarita Russo, Barbara Schiavo, Raffaella Schirò, Luciano Scotti, Angela Spandri, Maria Concetta Torrieri, Vittorio Vezzetti.

Ethics clearance: Institutional Ethics Committee Fondazione IRCCS Istituto Neurologico Carlo Besta's; Number 75 dated September 16, 2020.

Contributors: CDF, LDM, LM, MN, RS: data collection; AC: data analysis, drafting of the manuscript and study supervision; AC, MB: study supervision. All authors have designed the study concept, done critical revision of the manuscript for important intellectual content,approved the final version of manuscript, and are accountable for all aspects related to the study.

Funding: None; Competing interests: None stated.

Antonio Clavenna, ${ }^{1 *}$ Chiara Di Francesco, ${ }^{2}$ Lucia Di Maio, ${ }^{2}$ Laura Mauri, ${ }^{2}$ Mario Narducci, ${ }^{2}$ Raffaella Schirò, ${ }^{2}$ MaURIZIo Bonatr ${ }^{3}$ From 'Laboratory of Pharmacoepidemiology, Department of Public Health,

Istituto di Ricerche Farmacologiche Mario Negri IRCCS, Milan; ${ }^{2}$ Associazione Culturale Pediatri Milano; and ${ }^{3}$ Laboratory for Mother and Child Health, Istituto di Ricerche Farmacologiche Mario Negri IRCCS, Milan, Italy. *antonio.clavenna@marionegri.it

\section{REFERENCES}

1. Castagnoli R, Votto M, Licari A, et al. Severe acute respiratory syndrome coronavirus 2 (SARS-CoV-2) infection in children and adolescents: A systematic review. JAMA Pediatr. 2020; 174:882-9.

2. Mehta NS, Mytton OT, Mullins EWS, et al. SARS-CoV-2 (COVID-19): What do we know about children? A systematic review. Clin Infect Dis. 2020;71:2469-79.

3. Garazzino S, Lo Vecchio A, Pierantoni L, et al. Epidemiology, clinical features and prognostic factors of pediatric SARS-CoV-2 infection: Results from an italian multicenter study. Front Pediatr. 2021;9:649358.

4. Lazzerini M, Sforzi I, Trapani S, et al. Characteristics and risk factors for SARS-CoV-2 in children tested in the early phase of the pandemic: A cross-sectional study, Italy, 23 February to 24 May 2020. Euro Surveill. 2021;26:2001248.

5. Viner RM, Ward JL, Hudson LD, et al. Systematic review of reviews of symptoms and signs of COVID-19 in children and adolescents. Arch Dis Child. 2020 Dec 17; doi:archdischild-2020320972

6. Buonsenso D, Munblit D, De Rose C, et al. Preliminary evidence on long COVID in children. Acta Paediatr. 2021;110:2208-11.

7. Brackel CLH, Lap CR, Buddingh EP, et al. Pediatric longCOVID: An overlooked phenomenon? Pediatr Pulmonol. 2021;56:2495-2502.

8. Segre G, Campi R, Scarpellini F, et al. Interviewing children: The impact of the COVID-19 quarantine on children's perceived psychological distress and changes in routine. BMC Pediatr. 2021;21:231.

9. Ravens-Sieberer U, Kaman A, Erhart M, et al. Impact of the COVID-19 pandemic on quality of life and mental health in children and adolescents in Germany. Eur Child Adolesc Psychiatry. 2021;25:1-11.

10. Ford T, John A, Gunnell D. Mental health of children and young people during pandemic. BMJ. 2021;372: n614. 